OPEN ACCESS

Edited by:

Holger Rumpold,

Ordensklinikum Linz, Austria

Reviewed by:

Juan Liao,

Sichuan University, China

Antonio leni,

University of Messina, Italy

*Correspondence:

Dajun Deng

dengdajun@bjmu.edu.cn

Wenqiang Wei

weiwq@cicams.ac.cn

Specialty section:

This article was submitted to

Gastrointestinal Cancers,

a section of the journal

Frontiers in Oncology

Received: 22 March 2021

Accepted: 27 July 2021

Published: 17 August 2021

Citation:

Qin Y, Zhou J, Fan Z, Gu J, Li X, Lin D,

Deng $D$ and Wei W (2021) Evaluation of

the Impact of Intratumoral

Heterogeneity of Esophageal

Cancer on Pathological Diagnosis

and P16 Methylation and the

Representativity of Endoscopic Biopsy.

Front. Oncol. 11:683876.

doi: 10.3389/fonc.2021.683876

\section{Evaluation of the Impact of} Intratumoral Heterogeneity of Esophageal Cancer on Pathological Diagnosis and P16 Methylation and the Representativity of Endoscopic Biopsy

\author{
Yu Qin ${ }^{1}$, Jing Zhou ${ }^{2}$, Zhiyuan Fan ${ }^{1}$, Jianhua Gu ${ }^{1}$, Xinqing $L^{1}{ }^{1}$, Dongmei Lin $^{3}$, Dajun Deng ${ }^{2 *}$ \\ and Wenqiang Wei $^{1 *}$ \\ 1 National Cancer Registry Office, National Cancer Center/National Clinical Research Center for Cancer/Cancer Hospital, Chinese \\ Academy of Medical Sciences and Peking Union Medical College, Beijing, China, ${ }^{2}$ Key Laboratory of Carcinogenesis and \\ Translational Research, Peking University Cancer Hospital, Beijing, China, ${ }^{3}$ Department of Pathology, Peking University Cancer \\ Hospital, Beijing, China
}

Background: P16 methylation is expected to be potential diagnostic and therapeutic targets for esophageal cancer (EC). The intratumoral heterogeneity (ITH) of EC has been mentioned but has not been quantitatively measured yet. We aimed to clarify the impact of ITH on pathological diagnosis and P16 methylation, and the concordance between endoscopic biopsy and the corresponding surgically resected tissue.

Methods: We designed a systematic sampling method (SSM) compared with a general sampling method (GSM) to obtain EC tumor tissue, tumor biopsy, and normal squamous epithelium biopsy. MethyLight assay was utilized to test P16 methylation. All specimens obtained by the SSM were pathologically diagnosed.

Results: A total of 81 cases were collected by the GSM, and $91.4 \%$ and $8.6 \%$ of them were esophageal squamous cell carcinomas (ESCCs) and esophageal adenocarcinomas (EADs), respectively. Nine SSM cases were 100.0\% ESCCs. The positive rates of P16 methylation of the GSM tumor and normal tissues were 63.0\% (51/81) and 32.1\% (26/81), respectively. For SSM samples, tumor tissues were 100.0\% (40/40) EC and 85.0\% (34/40) P16 methylated; tumor biopsy was $64.4 \%$ (29/45) diagnosed of EC and $68.9 \%$ P16 methylated; the corresponding normal biopsies were 15.7\% (8/51) dysplasia and 54.9\% (28/51) P16 methylated. The concordance of pathological diagnosis and P16 methylation between tumor biopsy and the corresponding tumor tissue was $75.0 \%$ and $62.5 \%$, respectively.

Conclusion: The SSM we designed was efficient in measuring the ITH of EC. We found inadequate concordance between tumor biopsy and tissue in pathological diagnosis and P16 methylation.

Keywords: esophageal cancer, P16 methylation, intratumoral heterogeneity, endoscopic biopsy, representativity 


\section{INTRODUCTION}

Esophageal cancer (EC) is a fatal upper gastrointestinal malignant tumor. Approximately, there were 604,100 new EC cases and 544,076 deaths worldwide in 2020, ranking 7th and 6th in all cancer, respectively (1). The concealment of EC occurrence led to a poor clinical outcome of EC that associated with late diagnosis at advanced stage and therapy resistance, resulting in a poor 5-year survival rate of $30.3 \%$ (2). It is crucial to facilitate the EC precise diagnosis and therapy.

Recently, studies have characterized that the $\mathrm{CpG}$ island of the $P 16 / C D K N 2 A$ promoter is highly methylated in EC and might be a promising biomarker for EC personalized treatment and prognosis prediction (3-5). Since only a single sample was obtained to represent the methylation status of the whole tumor in present studies generally $(6,7)$, a wide variation of P16 methylated rate was noticed in different studies that based on EC tissues, $40.0 \%-90.0 \%$, which might be caused by ITH (intratumoral heterogeneity) that hindered its clinical application (8-14). Many studies uncovered the adverse effects of ITH on the cancer precise molecular classification, biomarker screening, and individualized precise treatment (15-17). Researches revealed that ITH can significantly affect the DNA methylation status of malignant tumors including breast cancer and lymphoma (15, 18). Since few studies focused on the impact of ITH on P16 methylation in EC $(6,19)$, how much it impacts on P16 methylation is still unclear.

In this study, we measured the P16 methylation level in EC and evaluated the impact of ITH on P16 methylation quantitatively to provide novel insights into the relationship between P16 methylation and EC.

\section{MATERIALS AND METHODS}

\section{Subject Enrollment}

In December 2019, EC inpatients who received esophagectomy and qualified the enrollment criteria were recruited from Linzhou Cancer Hospital, Henan province, a high incidence area of EC in China. Information including demographic characteristics, risk factors, and clinical diagnosis was collected.

\section{Enrollment Criteria}

1. Have not received radiotherapy or chemotherapy before operation.

2. In vitro time of surgical left tissue is less than an hour.

3. For the systematic sampling method, the length from the incisal edge to the tumor boundary that on the same side should be $3 \mathrm{~cm}$ at least.

\section{Human Research Subjects' Protection}

Written informed consent was obtained from all the participants. Ethics approval was provided by the Institutional Review Board of the Cancer Hospital of Chinese Academy of Medical sciences (No.15-151/1078).

\section{Sampling Method}

(1) General sampling method (GSM)

Obtain one piece of tissue at the tumor region randomly and one piece from the surgical margin using scalpel, respectively.

(2) Systematic sampling method (SSM)

A. See the esophagus tumor shape as a clock, and take mucosal biopsies at 3, 6, 9, and 12 o'clock and the center point using endoscopic biopsy forceps, respectively (Figure 1).

B. Obtain the paired tumor tissue sample right under the biopsied site using the forceps-biting marks as guides by a scalpel.

C. Biopsy from the normal surgical margin of tumor every centimeter.

All samples were store at $-80^{\circ} \mathrm{C}$.

\section{Pathological Diagnosis}

All samples were shipped to the laboratory at Peking University Cancer Hospital and Institute. Tissues obtained by the SSM were fixed with formalin solution and paraffin embedding and hematein eosin (H.E.) staining by experienced technologists. Histological slides were reviewed and diagnosed by senior pathologists from the Department of Pathology of Beijing Cancer Hospital.

\section{Genomic DNA Preparation and Bisulfite Conversion}

The frozen tissues obtained by the GSM were defrosted and grinded for genomic DNA extraction. For SSM samples, 8 to 10 pieces of $5-\mu \mathrm{m}$ paraffin-embedded tissue were cut for manual DNA extraction, and $50 \mu \mathrm{l}$ genomic DNA was eluted by Tris and EDTA (TE) buffer eventually.

Genomic DNA was modified by a DNA gold methylation kit (Zymo, Irvine, USA). Genomic DNA samples extracted from MGC803 and RKO cell lines were used as the P16 methylation negative and positive control, respectively.

\section{Real-Time qPCR Procedure}

We designed a useful and practical 115-bp MethyLight assay, reported previously, with very high specificity for the detection of P16 methylation clinically (20). The COL2A1 gene was selected as the internal reference gene. Gene sequences are listed below.

Methylated $P 16$ primer set: upstream $5^{\prime}$-cgcggtcgtggttagttagt- $3^{\prime}$ and downstream $5^{\prime}$-tacgctcgacgactacgaaa- $3^{\prime}$; methylated $P 16$ probe: 5'-6FAM-gttgtttttcgtcgtcggtt-TAMRA-3'; COL2A1 primer set: upstream $5^{\prime}$-tctaacaattataaactccaaccaccaa- $3^{\prime}$ and downstream $5^{\prime}$ gggaagatgggatagaagggaatat- $3^{\prime}$; COL $2 A 1$ probe: $5^{\prime}-6 \mathrm{FAM}$ ccttcattctaacccaatacctatcccacctctaaa-BHQ-1-3' Annealing temperature is $58.5^{\circ} \mathrm{C}$.

\section{Quality Control of qPCR Panel}

We set three replications for each sample. The following were the criteria for judging experimental results: the cycle threshold (CT) value is valid only if $\geq 2$ wells have a CT value, and the value is less than 40 for both. According to our previous study, we set 29.3 as the cutoff $\mathrm{CT}$ value of the internal reference gene COL2A1 for the 


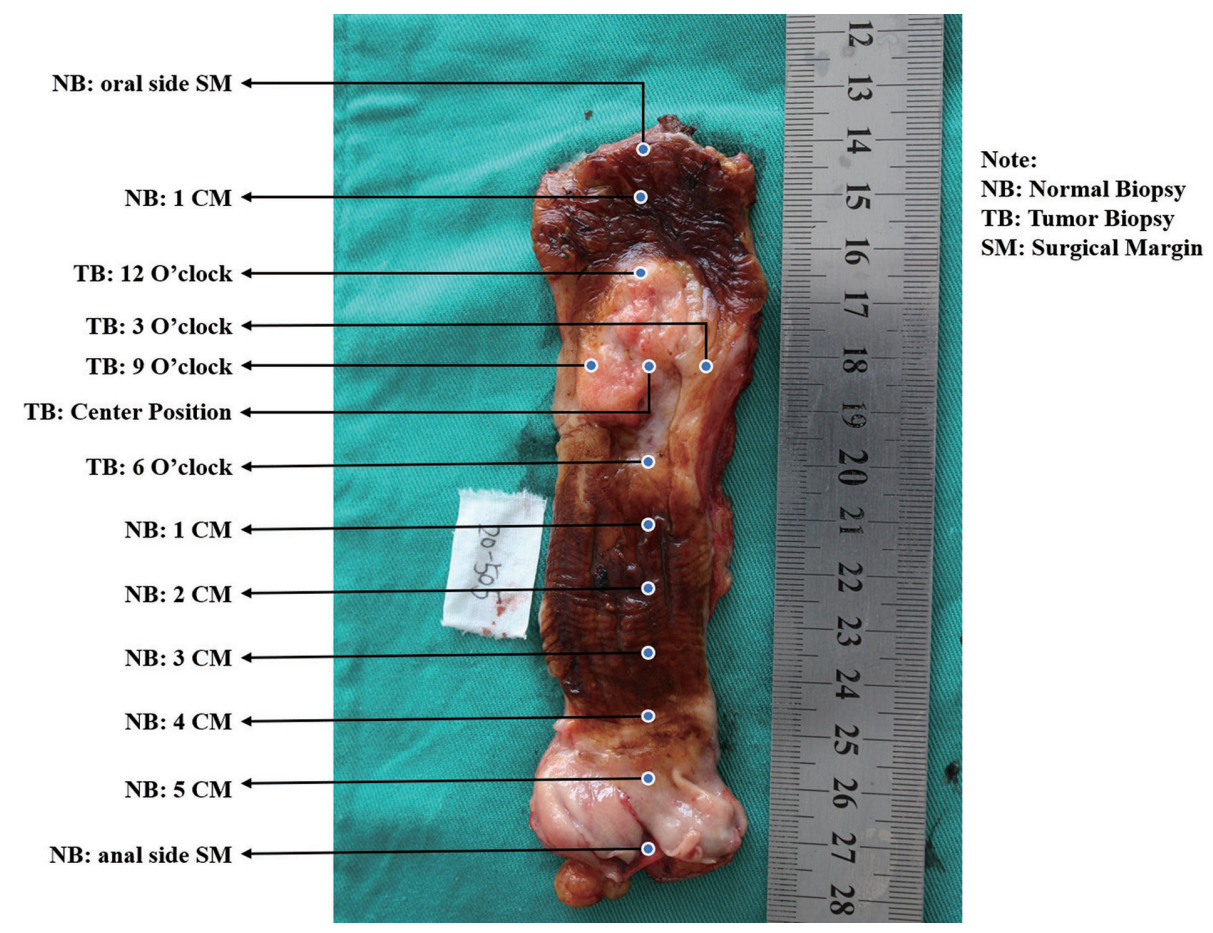

FIGURE 1 | Diagrammatic layout of systematic sampling method.

MethyLight assay result to reduce false negative (21). The COL2A1 gene CT value for MGC803 cells in a different panel was set equal to the first panel to eliminate the systematic bias.

\section{Statistical Analysis}

Demographic information such as age, gender, risk behaviors, and clinical characteristic was quantified. The positivity rates of P16 methylation between different subgroups were compared by using McNemar chi-square test. Paired Student's $t$ test was used to compare the difference of $\Delta \mathrm{Ct}$ values for P16 methylation between the tumor tissue and surgical margin tissues obtained by the GSM. Trend test was used in comparing the positive rates of P16 methylation between tumor tissues with different grade dysplasia. $P$ values less than 0.05 (two-sided) were statistically significant. Statistic Package for Social Science (SPSS) version 19.0 was used to analyze the data.

\section{RESULTS}

\section{General Characteristics of Participants and Sample Diagnosis}

Ninety EC patients were recruited, and their characteristics are shown in Table 1. Among the 81 cases collected by the GSM, 74 (91.4\%) were esophageal squamous cell carcinomas (ESCCs) and $7(8.6 \%)$ were esophageal adenocarcinomas (EADs), and all 9 cases obtained by the SSM were ESCCs. One SSM case failed to be obtained tumor tissue due to the thinness of tumor. In total,
TABLE 1 | General characteristics of participants.

\begin{tabular}{lc}
\hline Characteristics & $\mathbf{N},(\%)$ \\
\hline Age (Mean \pm SD) & $64.8 \pm 7.7$ \\
Gender & \\
Male & $71(78.9)$ \\
Female & $19(21.1)$ \\
Cigarette & \\
Yes & $54(60)$ \\
No & $36(40)$ \\
Alcohol & \\
Yes & $48(53.3)$ \\
No & $42(46.7)$ \\
Family history & \\
Yes & $62(68.9)$ \\
No & $28(31.1)$ \\
Pathological subtype & \\
ESCC & $74(91.4)$ \\
EAC & $7(8.6)$ \\
T stage & \\
1 b & $9(10.0)$ \\
2 & $16(17.8)$ \\
3 & $61(67.8)$ \\
$4 a$ & $4(4.4)$ \\
N stage & \\
0 & $39(43.3)$ \\
1 & $31(34.4)$ \\
2 & $13(14.4)$ \\
3 & $7(7.8)$ \\
M stage & \\
0 & $88(97.8)$ \\
1 & $2(2.2)$ \\
\hline ESC & \\
\hline
\end{tabular}

$\overline{E S C C}$, represents esophageal squamous cell carcinoma; EAC, represents esophageal adenocarcinoma. 
81 EC tumor tissues and the corresponding surgical margin tissues were collected by the GSM; 45 corresponding tumor biopsies (CTBs), 40 corresponding tumor tissues, and 51 corresponding surgical margin normal biopsies (CNBs) were collected by the SSM.

\section{P16 Methylation in GSM Samples}

Generally, 63.0\% (51/81) tumor tissues and 32.1\% (26/81) paired normal tissues were $P 16$ methylated; $75.6 \%$ tumor tissues from habitual drinkers were P16 methylated, which was significantly higher than those from nondrinkers $(p<0.05)$. For normal tissues, males had a higher $P 16$ methylation positive rate than females (38.1\% verse $11.1 \%$; $p<0.05)$. Patients at the advanced stage had a higher P16 methylation positive rate in both tumor and normal tissues than those at the early stage, though statistically insignificant (Table 2).

\section{Pathological Diagnosis of SSM Samples}

In the SSM sample set, 40 tumor samples were taken from 8 patients by typical pathologic sampling from surgical resected tumor mass/lesion under endoscope-biting sites. All these 40 samples were diagnosed as ESCC and thus used as the golden standard. However, for the 45 CTB biopsies taken from the same tumor set, only 29 (64.4\%) were diagnosed as ESCC, 10 (22.2\%) as high grade dysplasia (HGD), $5(11.1 \%)$ as moderate grade dysplasia (MGD), and $1(2.2 \%)$ as mild grade dysplasia (mGD), respectively, indicating an unavoidable false negative ESCC detection by individual endoscopic examinations/biopsies alone. In contrast, $51 \mathrm{CNB}$ biopsies from the corresponding surgical margins were diagnosed as follows: 0 ESCC, 2 HGDs, 2 MGDs,
4 mGDs, and 43 (84.3\%) normal epitheliums, indicating a very high specificity to detect ESCC by endoscopic examination (Table 3).

\section{P16 Methylation in SSM Samples}

In total, 68.9\% (31/45) tumor biopsies were P16 methylation positive, and no significant difference was found when comparing to the tumor tissues obtained by the GSM (63.0\%).

Around 58.3\% (21/36) and 88.9\% (8/9) tumor biopsies from the noncenter and center positions, respectively, were ESCC. For the noncenter position tumor biopsies, 69.4\% (25/36) were P16 methylated, and for the center position biopsies, $66.7 \%(6 / 9)$ were P16 methylated ( $p>0.1)$.

The $P 16$ methylation positive rate for tumor biopsies diagnosed of ESCC was $72.4 \%(21 / 29), 70.0 \%(7 / 10)$ for HGDs, and 50.0\% (3/6) for MGDs and mGDs, $(p$ trend $<0.05)$.

For all the above 40 ESCC tissues from 8 patients, $85.0 \%$ (34/ 40 ) were $P 16$ methylation positive while a $100.0 \%$ positive rate of P16 methylation was noticed in tumor tissues from the center position, which was higher than that in the tumor tissues obtained by the GSM $(63.0 \%$; $p<0.05)$.

Between tumor biopsy and corresponding tumor tissue samples, $75.0 \%(6 / 8)$ and $62.5 \%(5 / 8)$ patients showed inconsistent results of pathological diagnosis (Nos. 1, 3, 5, 7, 8, and 9) and $P 16$ methylation (Nos. 3, 4, 5, 8, and 9) (Table 4).

Among all the above surgical margin biopsies, $15.7 \%(8 / 51)$ were diagnosed of squamous epithelial dysplasia, including 2 HGDs, 2 MGDs, and 4 mGDs, and 6 dysplasia (2 HGDs, 2 MGDs, and $2 \mathrm{mGDs}$ ) located at $1 \mathrm{~cm}, 1 \mathrm{mGD}$ at $2 \mathrm{~cm}$, and $1 \mathrm{mGD}$ at $3 \mathrm{~cm}$ from the tumor mass/lesion (Table 5). Totally, $54.9 \%(28 / 51)$ of these CNB biopsies were P16 methylation

TABLE 2 | P16 methylation in GSM samples.

\begin{tabular}{|c|c|c|c|c|}
\hline & Tumor [N, (\%)] & $p$ & Normal [N, (\%)] & $p$ \\
\hline \multicolumn{5}{|l|}{ Gender } \\
\hline Female $(n=18)$ & $12(66.7)$ & 0.71 & $2(11.1)$ & $0.03^{*}$ \\
\hline \multicolumn{5}{|l|}{ Age } \\
\hline$\leq 59(n=16)$ & $8(50.0)$ & \multirow[t]{2}{*}{0.25} & $3(18.8)$ & \multirow[t]{2}{*}{0.43} \\
\hline $60-69(n=44)$ & 27 (61.4) & & $16(36.4)$ & \\
\hline Yes $(n=51)$ & 35 (68.6) & \multirow[t]{2}{*}{0.17} & 19 (37.3) & \multirow[t]{2}{*}{0.20} \\
\hline No $(n=30)$ & 16 (53.3) & & 7 (23.3) & \\
\hline \multicolumn{5}{|l|}{ Alcohol } \\
\hline Yes $(n=41)$ & $31(75.6)$ & \multirow[t]{2}{*}{$0.02^{*}$} & 16 (39.0) & \multirow[t]{2}{*}{0.18} \\
\hline No $(n=40)$ & $20(50.0)$ & & $10(25.0)$ & \\
\hline \multicolumn{5}{|l|}{ Family history } \\
\hline EAC & $5(71.4)$ & 0.94 & $3(42.9)$ & 0.83 \\
\hline \multicolumn{5}{|l|}{ Tumor stage } \\
\hline$T_{1-2}(n=23)$ & $13(56.5)$ & \multirow[t]{2}{*}{0.45} & $7(30.4)$ & \multirow[t]{2}{*}{0.84} \\
\hline$T_{3-4}(n=58)$ & $38(65.5)$ & & $19(32.8)$ & \\
\hline $\mathrm{N}_{0}(\mathrm{n}=36)$ & $21(58.3)$ & \multirow[t]{2}{*}{0.44} & $10(27.8)$ & \multirow[t]{2}{*}{0.46} \\
\hline$N_{1-3}(n=45)$ & $30(66.7)$ & & $16(35.6)$ & \\
\hline
\end{tabular}

*the difference is significant. 
TABLE 3 | The results of pathological diagnosis of all 136 SSM samples taken from 40 surgical ESCC mass samples.

\begin{tabular}{lccccc}
\hline Sample type & \multicolumn{3}{c}{ Pathological diagnosis (\%) } & Total \\
\cline { 2 - 5 } & Normal & mGD & MGD & HGD & ESCC \\
\hline Tumor tissue mass & 0 & 0 & 0 & 0 & 40 \\
CTB & 0 & $1(2.2)$ & $5(11.1)$ & $10(22.2)$ & $29(64.4)$ \\
CNB & $43(84.3)$ & $4(7.8)$ & $2(3.9)$ & $2(3.9)$ & 0 \\
\hline
\end{tabular}

CTB, corresponding tumor biopsy; CNB, corresponding normal biopsy.

positive, consisting of 17 (60.7\%) from $1-2 \mathrm{~cm}, 9$ (32.1\%) from $3-4 \mathrm{~cm}$, and $2(7.1 \%)$ from $5-6 \mathrm{~cm}$, respectively $(p$ trend $<0.05)$ (Table 5). In “1-5 cm” subgroups, $91.7 \%, 50.0 \%, 50.0 \%, 30.0 \%$, and $25.0 \%$ biopsies were $P 16$ methylation positive, respectively, showing a decreasing trend with the increase in the distance from the biopsy site to the tumor mass/lesion $\left(p_{\text {trend }}<0.05\right)$.

\section{DISCUSSION}

Due to the unfavorable prognosis of advanced EC, a number of studies hoped to seek biomarkers that have a diagnostic value in the early stage of EC (19). P16 methylation was found prevalent in EC tissues and demonstrated a promising diagnostic, therapeutic, and prognostic value $(3,5,22-24)$. However, many studies have shown large variations in detecting P16 methylation with a positive rate ranging from $45 \%$ to $88 \%$, even in the same race and same sites (25-27). Researchers had already recognized the variation in EC and speculated that it might be influenced by ITH on tumor suppressor methylation $(6,7,28)$.

In this study, we designed a systematic sampling method to measure the ITH quantitatively and the representativeness of tumor biopsy on pathological diagnosis and P16 methylation.
We found a positive rate of $63.0 \%$ on $P 16$ methylation in the tumor tissues that were obtained by the GSM, which is at the same level as others' reports $(27,29)$. The positive rate of P16 methylation in nonposition-specific and center tumor tissue was $85.0 \%$ and $100.0 \%$, respectively, which was significantly higher than that in the GSM samples. This suggests that the tumor tissue from the center position was the most representative of the whole tumor lesion compared to other positions. Thus, the ITH might influence the positive rate of $P 16$ methylation if only one piece of tumor tissue was collected randomly $(19,28,30)$. Our current study recommends that tumor tissue should be obtained at the center of the EC tumor rather than other position in future studies. Other researchers also revealed it in EC and other malignant tumor including breast cancer, liver cancer, colorectal cancer, and Ewing sarcoma (15-18).

We also noticed P16 methylation prevalent in the paired surgical margin obtained from EC patients, which was consistent with others' studies $(31,32)$, revealing that $P 16$ methylation has already occurred and developed in the "normal" tissue, and the surgical margin is not the most appropriate blank control in EC biomarker screening (5).

The tumor biopsy specimen was commonly analyzed in a number of studies, especially in studies of EC screening and early

TABLE 4 | Diagnosis and p16 methylation status of 40 tumor tissues and 45 tumor biopsies.

\begin{tabular}{|c|c|c|c|c|c|c|c|c|c|c|c|}
\hline \multirow[t]{3}{*}{ Patients No. } & \multirow[t]{3}{*}{ Sample type } & \multicolumn{10}{|c|}{ Position (O'clock) } \\
\hline & & \multicolumn{2}{|c|}{3} & \multicolumn{2}{|c|}{6} & \multicolumn{2}{|c|}{9} & \multicolumn{2}{|c|}{12} & \multicolumn{2}{|c|}{ Center } \\
\hline & & Diag & P16m & Diag & P16m & Diag & P16m & Diag & P16m & Diag & P16m \\
\hline \multirow[t]{2}{*}{1} & TB & ESCC & + & ESCC & + & HGD & + & ESCC & + & ESCC & + \\
\hline & $\Pi$ & ESCC & + & ESCC & + & ESCC & + & ESCC & + & ESCC & + \\
\hline \multirow[t]{2}{*}{2} & TB & ESCC & + & ESCC & - & ESCC & + & ESCC & + & ESCC & + \\
\hline & $\pi$ & ESCC & + & ESCC & - & ESCC & + & ESCC & + & ESCC & + \\
\hline \multirow[t]{2}{*}{3} & TB & ESCC & + & HGD & + & HGD & - & ESCC & - & ESCC & + \\
\hline & $\pi$ & ESCC & + & ESCC & + & ESCC & + & ESCC & + & ESCC & + \\
\hline \multirow[t]{2}{*}{4} & TB & ESCC & + & ESCC & - & ESCC & - & ESCC & - & ESCC & + \\
\hline & $\pi$ & ESCC & - & ESCC & - & ESCC & + & ESCC & - & ESCC & + \\
\hline \multirow[t]{2}{*}{5} & TB & ESCC & + & ESCC & + & HGD & + & HGD & + & HGD & - \\
\hline & $\Pi \pi$ & ESCC & - & ESCC & + & ESCC & + & ESCC & + & ESCC & + \\
\hline \multirow[t]{2}{*}{6} & TB & ESCC & + & ESCC & + & MGD & - & MGD & + & EC & - \\
\hline & $\pi$ & NA & NA & NA & NA & NA & NA & NA & NA & NA & NA \\
\hline \multirow[t]{2}{*}{7} & TB & MGD & + & ESCC & + & HGD & + & MGD & + & ESCC & + \\
\hline & $\pi$ & ESCC & + & ESCC & + & ESCC & + & ESCC & + & ESCC & + \\
\hline \multirow[t]{2}{*}{8} & TB & mGD & + & MGD & - & MGD & - & ESCC & + & ESCC & - \\
\hline & $\pi$ & ESCC & + & ESCC & + & ESCC & - & ESCC & + & ESCC & + \\
\hline \multirow[t]{2}{*}{9} & TB & HGD & - & ESCC & + & ESCC & - & ESCC & + & ESCC & + \\
\hline & $\Pi \pi$ & ESCC & + & ESCC & + & ESCC & + & ESCC & + & ESCC & + \\
\hline
\end{tabular}

TB, represents tumor biopsy; $\pi$, represents tumor tissue. + , represents $p 16$ methylated; -, represents p16 un-methylated. 
TABLE 5 | Pathological diagnosis and p16 methylation of SM biopsies.

\begin{tabular}{|c|c|c|c|c|c|c|c|c|}
\hline No. & Direction & & \multicolumn{6}{|c|}{ The distance to the tumor border $(\mathrm{CM})^{\mathrm{a}}$} \\
\hline 1 & O & Diag & MGD & $\mathrm{N}$ & $\mathrm{N}$ & $\mathrm{N}$ & N & \\
\hline \multirow[t]{2}{*}{2} & O & Diag & HGD & N & $m G D$ & N & N & $\mathrm{N}$ \\
\hline & & $\mathrm{P} 16 \mathrm{~m}$ & + & - & + & - & + & + \\
\hline \multirow[t]{2}{*}{3} & 0 & Diag & HGD & $\mathrm{N}$ & $\mathrm{N}$ & $\mathrm{N}$ & & \\
\hline & & $\mathrm{P} 16 \mathrm{~m}$ & + & - & - & - & & \\
\hline \multirow[t]{4}{*}{4} & 0 & Diag & $\mathrm{N}$ & $\mathrm{N}$ & $\mathrm{N}$ & & & \\
\hline & & P16m & + & + & + & & & \\
\hline & $A$ & Diag & $\mathrm{N}$ & $\mathrm{N}$ & $\mathrm{N}$ & $\mathrm{N}$ & N & \\
\hline & & P16m & + & + & - & - & - & \\
\hline 5 & 0 & Diag & $\mathrm{N}$ & $\mathrm{N}$ & $\mathrm{N}$ & & & \\
\hline \multirow{2}{*}{7} & A & Diag & $\mathrm{N}$ & $\mathrm{N}$ & $\mathrm{N}$ & $\mathrm{N}$ & & \\
\hline & & $\mathrm{P} 16 \mathrm{~m}$ & + & + & + & + & & \\
\hline \multirow[t]{2}{*}{8} & 0 & Diag & $m G D$ & $N$ & $\mathrm{~N}$ & $\mathrm{~N}$ & & \\
\hline & & $\mathrm{P} 16 \mathrm{~m}$ & + & - & + & + & & \\
\hline \multirow[t]{2}{*}{9} & O & Diag & $m G D$ & $N$ & $\mathrm{~N}$ & $\mathrm{~N}$ & & \\
\hline & & $\mathrm{P} 16 \mathrm{~m}$ & + & - & - & - & & \\
\hline
\end{tabular}

+ , represents $p 16$ methylated; -, represents $p 16$ un-methylated. $\mathrm{N}$, represents normal. ${ }^{a} T$ The last sample of each row was obtained from the incisal edge. O, represents the oral edge and $A$, represents the anal edge.

diagnosis, to represent whole individual cases $(33,34)$. However, researchers found that one single biopsy did not represent tumor or lesions adequately. On the aspect of pathological diagnosis, our results revealed that more than a third (35.6\%) of biopsies obtained from the EC tumor surface were diagnosed as non-EC. A significant pathological difference between noncenter and center biopsies was found ( $58.3 \%$ vs. $88.9 \%)$. There were two patients that could be pathologically diagnosed as EC by using four biopsies from different position at least, suggesting that inadequate diagnosis (false negative) could occur easily when pathological diagnosis was made based on only one biopsy. For P16 methylation, a lower positive rate of $68.9 \%$ was found in tumor biopsies compared to tumor tissues (85.0\%). Although a P16 methylation positive rate between nonspecific position, clock position, and center position was insignificantly different, a positive correlation was found between the P16 methylation rate and the severity of dysplasia lesions, which was consistent with other researchers' reports (35). Since one-third of the biopsies were $P 16$ methylation negative even from the center position, we considered that the position of tumor biopsy scarcely influenced the detection of P16 methylation. Nonetheless, a smaller number of biopsies might result in a higher false negative.

Among the 40 pairs of tumor biopsies and corresponding tissues, $25.0 \%$ were inconsistent on P16 methylation results in total, and with a kappa value of 0.31 , had shown a lowly concordant between the biopsy and surgically resected specimens from the same patients. Taking the P16 methylation result for tumor tissues as the golden standard, a false negative rate of $23.5 \%$ and a false positive rate of $33.3 \%$ were noticed in tumor biopsies. However, Ken Hatogai et al. found that highly concordant between endoscopic biopsy and surgically resected specimens from the same EC patients in detecting PIK3CA mutation status with an overall concordance rate of $98.3 \%$ $(178 / 181)$ (36), which is inconsistent with our results.

The esophageal squamous epithelium region between the tumor area and the surgical margin was considered as autologous blank control in many studies usually. However, some researchers discovered that the P16 methylation might be positive even in a histologically normal sample of EC patients, with positive rates of $36.8 \%$ (32) and $38.3 \%$ (37), which is consistent with the rate of $33.3 \%$ in our study. A lower positive rate, ranging from $0.0 \%$ to $21.3 \%$, was also found by other researchers $(26,38)$. Our results suggested that this controversial finding might be induced by the length of the esophageal squamous epithelium region and the normal tissue position. It is worth noting that the positive rate of $P 16$ methylation increased to approximately $60.0 \%$ suddenly if the biopsy position moved forward just $1 \mathrm{~cm}$ to the surgical margin, and it remains at a lower positive rate of P16 methylation only when it reaches over $3 \mathrm{~cm}$ from the tumor lesion. This might result in an uncanny false positive rate and confuse other researchers. Therefore, when an autologous blank control is essential, a normal tissue should be obtained at a position $3 \mathrm{~cm}$ far from the tumor lesion if conditions permit.

There were several limitations in this study. Primarily, the sample size is small, and the EC patients were heterogenous, which reduced the reliability of our findings and caused a limited 
extend power. This is a pilot study on the representativity of pathological diagnosis and DNA methylation of tumor suppressors applied on EC screening. We will validate the results in a larger cohort in the future. Secondly, we tested P16 methylation only, and the heterogeneity status of other tumor suppressors is still not clear yet.

\section{CONCLUSION}

Significant impacts of ITH on pathological diagnosis and P16 methylation of EC and the concordance between tumor tissue and biopsy samples were observed in our SSM analyses Several suggestions about proper sampling to improve the performance of endoscope examination/biopsy were recommended. More studies with a larger sample size, more biomarkers, and highresolution methylation testing are needed to be implemented in a larger cohort to evaluate the sampling method in the future.

\section{DATA AVAILABILITY STATEMENT}

The raw data supporting the conclusions of this article will be made available by the authors, without undue reservation.

\section{ETHICS STATEMENT}

The studies involving human participants were reviewed and approved by the Institutional Review Board of the Cancer

\section{REFERENCES}

1. Siegel RL, Miller KD, Fuchs HE, Jemal A. Cancer Statistics, 2021. CA: A Cancer J Clin (2021) 71(1):7-33. doi: 10.3322/caac.21654

2. Zeng H, Chen W, Zheng R, Zhang S, Ji JS, Zou X, et al. Changing Cancer Survival in China During 2003-15: A Pooled Analysis of 17 Population-Based Cancer Registries. Lancet Glob Health (2018) 6(5):e555-67. doi: 10.1016/ S2214-109X(18)30127-X

3. Pu W, Wang C, Chen S, Zhao D, Zhou Y, Ma Y, et al. Targeted Bisulfite Sequencing Identified a Panel of DNA Methylation-Based Biomarkers for Esophageal Squamous Cell Carcinoma (ESCC). Clin Epigenet (2017) 9:129. doi: 10.1186/s13148-017-0430-7

4. Nagata H, Kozaki KI, Muramatsu T, Hiramoto H, Tanimoto K, Fujiwara N, et al. Genome-Wide Screening of DNA Methylation Associated With Lymph Node Metastasis in Esophageal Squamous Cell Carcinoma. Oncotarget (2017) 8(23):37740-50. doi: 10.18632/oncotarget.17147

5. Ma K, Cao B, Guo M. The Detective, Prognostic, and Predictive Value of DNA Methylation in Human Esophageal Squamous Cell Carcinoma. Clin Epigenet (2016) 8:43. doi: 10.1186/s13148-016-0210-9

6. Hao JJ, Lin DC, Dinh HQ, Mayakonda A, Jiang YY, Chang C, et al. Spatial Intratumoral Heterogeneity and Temporal Clonal Evolution in Esophageal Squamous Cell Carcinoma. Nat Genet (2016) 48(12):1500-7. doi: 10.1038/ ng. 3683

7. Lin L, Lin DC. Biological Significance of Tumor Heterogeneity in Esophageal Squamous Cell Carcinoma. Cancers (Basel) (2019) 11(8):1156. doi: 10.3390/ cancers11081156

8. Das M, Saikia BJ, Sharma SK, Sekhon GS, Mahanta J, Phukan RK. P16 Hypermethylation: A Biomarker for Increased Esophageal Cancer Susceptibility in High Incidence Region of North East India. Tumour Biol (2015) 36(3):1627-42. doi: 10.1007/s13277-014-2762-7
Hospital of Chinese Academy of Medical Sciences. The patients/participants provided their written informed consent to participate in this study.

\section{AUTHOR CONTRIBUTIONS}

YQ, WW, and DD contributed to the conception and design of the study and wrote the manuscript. YQ, JZ, WW, and DD interpreted the results, literature search, and construction of tables and figures. DL reviewed the pathological slides. YQ, JG, and XL contributed to questionnaire information and specimen collection. All authors contributed to the article and approved the submitted version.

\section{FUNDING}

This study is supported by the National Key R\&D Program of China (2016YFC0901400, 2016YFC0901404) and the National Natural Science Foundation of China (81974493).

\section{ACKNOWLEDGMENTS}

The authors would like to thank Drs. Guanggen Jiao, Yanzeng Hao, Jiangying Yue, and Mr. Xinqi Liu from Yaocun Esophageal Cancer Hospital for help in recruiting subjects and collecting clinical information and specimens in this study.

9. Xu R, Wang F, Wu L, Wang J, Lu C. A Systematic Review of Hypermethylation of P16 Gene in Esophageal Cancer. Cancer Biomark (2013) 13(4):215-26. doi: 10.3233/CBM-130355

10. Xing EP, Nie Y, Song Y, Yang GY, Cai YC, Wang LD, et al. Mechanisms of Inactivation of p14ARF, p15INK4b, and p16INK4a Genes in Human Esophageal Squamous Cell Carcinoma. Clin Cancer Res (1999) 5 (10):2704-13.

11. Abbaszadegan MR, Raziee HR, Ghafarzadegan K, Shakeri MT, Afsharnezhad S, Ghavamnasiry MR. Aberrant P16 Methylation, a Possible Epigenetic Risk Factor in Familial Esophageal Squamous Cell Carcinoma. Int J Gastrointest Cancer (2005) 36(1):47-54. doi: 10.1385/ IJGC:36:1:047

12. Tokugawa T, Sugihara H, Tani T, Hattori T. Modes of Silencing of P16 in Development of Esophageal Squamous Cell Carcinoma. Cancer Res (2002) 62 (17):4938-44.

13. Chen W, Yang C, Yang L, Qi C, Tian S, Han Y, et al. Association of Roasting Meat Intake With the Risk of Esophageal Squamous Cell Carcinoma of Kazakh Chinese via Affecting Promoter Methylation of P16 Gene. Asia Pac J Clin Nutr (2014) 23(3):488-97. doi: 10.6133/apjen.2014.23.3.11

14. Chen J, Huang ZJ, Duan YQ, Xiao XR, Jiang JQ, Zhang R. Aberrant DNA Methylation of P16, MGMT, and hMLH1 Genes in Combination With MTHFR C677T Genetic Polymorphism and Folate Intake in Esophageal Squamous Cell Carcinoma. Asian Pac J Cancer Prev (2012) 13(10):5303-6. doi: 10.7314/APJCP.2012.13.10.5303

15. Byler S, Goldgar S, Heerboth S, Leary M, Housman G, Moulton K, et al. Genetic and Epigenetic Aspects of Breast Cancer Progression and Therapy. Anticancer Res (2014) 34(3):1071-7.

16. Liu J, Dang H, Wang XW. The Significance of Intertumor and Intratumor Heterogeneity in Liver Cancer. Exp Mol Med (2018) 50(1):e416. doi: 10.1038/ emm.2017.165 
17. Jones HG, Jenkins G, Williams N, Griffiths P, Chambers P, Beynon J, et al. Genetic and Epigenetic Intra-Tumour Heterogeneity in Colorectal Cancer. World J Surg (2017) 41(5):1375-83. doi: 10.1007/s00268-016-3860-z

18. Sheffield NC, Pierron G, Klughammer J, Datlinger P, Schonegger A, Schuster M, et al. DNA Methylation Heterogeneity Defines a Disease Spectrum in Ewing Sarcoma. Nat Med (2017) 23(3):386-95. doi: 10.1038/ nm. 4273

19. Lin DC, Wang MR, Koeffler HP. Genomic and Epigenomic Aberrations in Esophageal Squamous Cell Carcinoma and Implications for Patients. Gastroenterology (2018) 154(2):374-89. doi: 10.1053/j.gastro.2017.06.066

20. Zhou J, Cao J, Lu Z, Liu H, Deng D. A 115-bp MethyLight Assay for Detection of p16 (CDKN2A) Methylation as a Diagnostic Biomarker in Human Tissues. BMC Med Genet (2011) 12:67. doi: 10.1186/1471-2350-12-67

21. Liu Z, Zhou J, Gu L, Deng D. Significant Impact of Amount of PCR Input Templates on Various PCR-Based DNA Methylation Analysis and Countermeasure. Oncotarget (2016) 7(35):56447-55. doi: 10.18632/ oncotarget.10906

22. Lu T, Chen D, Wang Y, Sun X, Li S, Miao S, et al. Identification of DNA Methylation-Driven Genes in Esophageal Squamous Cell Carcinoma: A Study Based on The Cancer Genome Atlas. Cancer Cell Int (2019) 19:52. doi: 10.1186/s12935-019-0770-9

23. Li D, Zhang L, Liu Y, Sun H, Onwuka JU, Zhao Z, et al. Specific DNA Methylation Markers in the Diagnosis and Prognosis of Esophageal Cancer. Aging (Albany NY) (2019) 11(23):11640-58. doi: 10.18632/aging.102569

24. Chen Y, Liao LD, Wu ZY, Yang Q, Guo JC, He JZ, et al. Identification of Key Genes by Integrating DNA Methylation and Next-Generation Transcriptome Sequencing for Esophageal Squamous Cell Carcinoma. Aging (Albany NY) (2020) 12(2):1332-65. doi: 10.18632/aging.102686

25. Taghavi N, Biramijamal F, Sotoudeh M, Khademi H, Malekzadeh R, Moaven O, et al. p16INK4a Hypermethylation and p53, p16 and MDM2 Protein Expression in Esophageal Squamous Cell Carcinoma. BMC Cancer (2010) 10:138. doi: 10.1186/1471-2407-10-138

26. Li B, Wang B, Niu LJ, Jiang L, Qiu CC. Hypermethylation of Multiple TumorRelated Genes Associated With DMNT3b Upregulation Served as a Biomarker for Early Diagnosis of Esophageal Squamous Cell Carcinoma. Epigenetics (2011) 6(3):307-16. doi: 10.4161/epi.6.3.14182

27. Wang CC, Mao WM, Ling ZQ. DNA Methylation Status of RARbeta2 and p16(INK4alpha) in Peripheral Blood and Tumor Tissue in Patients With Esophageal Squamous Cell Carcinoma. Zhonghua Zhong Liu Za Zhi (2012) 34 (6):441-5. doi: 10.3760/cma.j.issn.0253-3766.2012.06.009

28. Guo M, Peng Y, Gao A, Du C, Herman JG. Epigenetic Heterogeneity in Cancer. Biomark Res (2019) 7:23. doi: 10.1186/s40364-019-0174-y

29. Wang J, Sasco AJ, Fu C, Xue H, Guo G, Hua Z, et al. Aberrant DNA Methylation of P16, MGMT, and hMLH1 Genes in Combination With MTHFR C677T Genetic Polymorphism in Esophageal Squamous Cell Carcinoma. Cancer Epidemiol Biomarkers Prev (2008) 17(1):118-25. doi: 10.1158/1055-9965.EPI-07-0733

30. Ling S, Hu Z, Yang Z, Yang F, Wu CI. Extremely High Genetic Diversity in a Single Tumor Points to Prevalence of Non-Darwinian Cell Evolution. Proc Natl Acad Sci (2015) 112(47):E6496-505. doi: 10.1073/pnas.1519556112
31. Yamashita S, Kishino T, Takahashi T, Shimazu T, Charvat H, Kakugawa Y, et al. Genetic and Epigenetic Alterations in Normal Tissues Have Differential Impacts on Cancer Risk Among Tissues. Proc Natl Acad Sci USA (2018) 115 (6):1328-33. doi: 10.1073/pnas.1717340115

32. Gonzaga IM, Soares Lima SC, Nicolau MC, Nicolau-Neto P, da Costa NM, de Almeida Simao T, et al. TFF1 Hypermethylation and Decreased Expression in Esophageal Squamous Cell Carcinoma and Histologically Normal Tumor Surrounding Esophageal Cells. Clin Epigenet (2017) 9:130. doi: 10.1186/ s13148-017-0429-0

33. Wei WQ, Chen ZF, He YT, Feng H, Hou J, Lin DM, et al. Long-Term FollowUp of a Community Assignment, One-Time Endoscopic Screening Study of Esophageal Cancer in China. J Clin Oncol (2015) 33(17):1951-7. doi: 10.1200/ JCO.2014.58.0423

34. Cao J, Zhou J, Gao Y, Gu L, Meng H, Liu H, et al. Methylation of P16 CpG Island Associated With Malignant Progression of Oral Epithelial Dysplasia: A Prospective Cohort Study. Clin Cancer Res (2009) 15(16):5178-83. doi: 10.1158/1078-0432.CCR-09-0580

35. Guo M, Ren J, House MG, Qi Y, Brock MV, Herman JG. Accumulation of Promoter Methylation Suggests Epigenetic Progression in Squamous Cell Carcinoma of the Esophagus. Clin Cancer Res (2006) 12(15):4515-22. doi: 10.1158/1078-0432.CCR-05-2858

36. Hatogai K, Fujii S, Kojima T, Daiko H, Doi T, Ohtsu A, et al. Concordance Between PIK3CA Mutations in Endoscopic Biopsy and Surgically Resected Specimens of Esophageal Squamous Cell Carcinoma. BMC Cancer (2017) 17 (1):36. doi: 10.1186/s12885-016-3041-3

37. Lu C, Xie H, Wang F, Shen H, Wang J. Diet Folate, DNA Methylation and Genetic Polymorphisms of MTHFR C677T in Association With the Prognosis of Esophageal Squamous Cell Carcinoma. BMC Cancer (2011) 11:91. doi: 10.1186/1471-2407-11-91

38. Wang F, Xie XJ, Piao YS, Liu B, Wang LD. Methylation of P16 and Hmlh1 Genes in Esophageal Squamous Cell Carcinoma and Reflux Esophagitis. Zhonghua Bing Li Xue Za Zhi (2011) 40(8):537-41. doi: 10.3760/ cma.j.issn.0529-5807.2011.08.009

Conflict of Interest: The authors declare that the research was conducted in the absence of any commercial or financial relationships that could be construed as a potential conflict of interest.

Publisher's Note: All claims expressed in this article are solely those of the authors and do not necessarily represent those of their affiliated organizations, or those of the publisher, the editors and the reviewers. Any product that may be evaluated in this article, or claim that may be made by its manufacturer, is not guaranteed or endorsed by the publisher.

Copyright $\odot 2021$ Qin, Zhou, Fan, Gu, Li, Lin, Deng and Wei. This is an open-access article distributed under the terms of the Creative Commons Attribution License (CC BY). The use, distribution or reproduction in other forums is permitted, provided the original author(s) and the copyright owner(s) are credited and that the original publication in this journal is cited, in accordance with accepted academic practice. No use, distribution or reproduction is permitted which does not comply with these terms. 